OPEN ACCESS

Edited by:

Mirta Fiorio,

University of Verona, Italy

Reviewed by:

Davide Martino,

King's College London, United Kingdom

Marta Bortoletto,

Centro San Giovanni di Dio

Fatebenefratelli (IRCCS), Italy

*Correspondence:

Jason L. He

jason.he@deakin.edu.au

Specialty section:

This article was submitted to

Movement Disorders,

a section of the journal

Frontiers in Neurology

Received: 13 December 2017 Accepted: 07 March 2018

Published: 23 March 2018

Citation:

He JL, Fuelscher I, Enticott PG, Teo W-p, Barhoun P and Hyde C (2018) Interhemispheric Cortical Inhibition Is Reduced in Young Adults With Developmental Coordination Disorder.

Front. Neurol. 9:179. doi: 10.3389/fneur.2018.00179

\section{Interhemispheric Cortical Inhibition Is Reduced in Young Adults With Developmental Coordination Disorder}

\author{
Jason L. He ${ }^{1 *}$, Ian Fuelscher ${ }^{1}$, Peter G. Enticott', Wei-peng Teo ${ }^{2}$, Pamela Barhoun ${ }^{1}$ \\ and Christian Hyde ${ }^{1}$ \\ 'Deakin Child Study Centre, School of Psychology, Deakin University, Geelong, VIC, Australia, ${ }^{2}$ School of Exercise \\ and Nutrition Sciences, Institute for Physical Activity and Nutrition (IPAN), Deakin University, Geelong, VIC, Australia
}

Introduction: While the etiology of developmental coordination disorder (DCD) is yet to be established, brain-behavior modeling provides a cogent argument that neuropathology may subserve the motor difficulties typical of DCD. We argue that a number of the core behavioral features of the DCD profile (such as poor surround inhibition, compromised motor inhibition, and the presence of mirror movements) are consistent with difficulties regulating inhibition within the primary motor cortex (M1). This study aimed to be the first account of the integrity of cortical inhibition in motor cortices in DCD.

Method: The sample consisted of eight adults with DCD aged (18-30 years) and 10 aged matched neurotypical controls. Participants received a common battery of single and paired-pulse transcranial magnetic stimulation from which a series of neurophysiological measures classically used to measure intra- [e.g., short-interval cortical inhibition (SICI), long-interval cortical inhibition (LICI), and cortical silent period] and inter hemispheric [e.g., ipsilateral silent period (ISP)] cortical inhibition of the M1 at rest were recorded.

Results: While no group differences were observed for any measure of intrahemispheric cortical inhibition, individuals with DCD demonstrated significantly reduced interhemispheric cortical inhibition relative to controls, shown by consistently lower ISP ratios.

Conclusion: Our findings are consistent with the view that regulation of cortical inhibition of M1 activity may be atypical in individuals with DCD, indicating differential GABAergic operation. This effect, however, appears to be select to cortical inhibition. Importantly, our data support the notion that reduced interhemispheric M1 cortical inhibition may at least partly explain commonly reported difficulties with bimanual motor control in DCD. The neurochemical implications and limitations of this evidence will be discussed.

Keywords: developmental coordination disorder, motor control, cortical inhibition, transcranial magnetic stimulation, movement disorders, movement, interhemispheric connectivity 


\section{RESEARCH HIGHLIGHTS}

- A number of core behavioral features of DCD are consistent with difficulties regulating GABAergic activity (viz cortical inhibition) within the primary motor cortex (M1).

- This study used TMS to probe the integrity of cortical inhibition in the M1 in individuals with DCD.

- While intrahemispheric M1 cortical inhibition appears to be preserved in DCD, interhemispheric M1 cortical inhibition was significantly reduced relative to typically developing individuals.

- Our study is the first to provide evidence that regulation of GABAergic activity within the M1 may be atypical in DCD.

\section{INTRODUCTION}

Developmental coordination disorder (DCD) is characterized by atypical development and performance of motor skill in the absence of any identifiable neurological or medical conditions (1). Diagnosed individuals present with motor skills substantially below that expected given their age, limiting their ability to perform daily tasks requiring coordinated movements [e.g., using utensils, playing sports, and self-care $(2,3)]$. Motor difficulties symptomatic of DCD emerge in childhood, with the syndrome diagnosed early in development, typically during the primary school years (4). Initially theorized to result from a delay in brain development [e.g., minimal brain dysfunction: see (5) for a good review], the primary symptoms of DCD were expected to dissipate with age (6). It is now well understood that the motor skill difficulties seen in DCD persist into adulthood for a substantial proportion of diagnosed individuals (7-9), as do the secondary psychosocial difficulties $(10,11)$. These findings highlight the importance of early identification of DCD and the need to develop age-appropriate assessment and therapeutic strategies.

While the etiology of DCD remains unclear, brain-behavior modeling provides a cogent argument that cortical neuropathology may subserve atypical motor development $(5,12-14)$. Indeed, in vivo neurological measurement of individuals with DCD has increased substantially over the last decade (14). These studies have primarily focused on the pattern of cortical de/activation (via fMRI or EEG) when individuals with DCD perform perceptuo-motor and cognitive tasks, or the structural integrity of motor circuitry (via MRI). While promising, these data remain sparse and inconsistent. Accordingly, the "neural signature" of DCD is yet to be established (5), and further work is required. In this study, we investigate whether atypical regulation of the nervous system's principle inhibitory neurotransmitter, gamma-aminobutyric acid (GABA), within the primary motor cortex (M1) may explain some of the core features of the DCD symptom profile (see below). To the best of our knowledge, no study has directly tested this important hypothesis nor, have the neurochemical properties of poor motor control in DCD been reported on. Since regulation of M1 activity has been shown to be amenable to therapeutic intervention in populations with and without motor impairment (15-18), clarifying this issue holds considerable therapeutic significance for individuals with DCD.

\section{Primary Motor Cortical Inhibition Is Fundamental to Motor Control}

The M1 is the final relay point for outgoing motor commands before they descend the spinal cord, receiving input from other motor cortical and subcortical structures (19). The role of M1 in producing movement is well established, with excitation increasing within the contralateral M1 immediately (i.e., $\approx 120 \mathrm{~ms}$ ) before activation of task-relevant peripheral muscles $(19,20)$. However, it is becoming increasingly clear that inhibitory mechanisms within the M1 are equally as important to producing mature movement (19-21). That is, accurate motor output from the M1 is part the product of excitatory commands supporting the movement of choice and part inhibitory commands suppressing undesired muscle activity (22).

Intracortically, inhibitory interneurons within the contralateral M1 are involved in the suppression of proximal, task-inappropriate muscles during voluntary manual movement (21, 23-26). Specifically, before (and during) unimanual movement, GABAergic activity decreases for task-relevant muscles, yet simultaneously and selectively increases for nearby task-irrelevant or synergistic abductors (27-29). This so-called "surround inhibition" is touted to be the putative neurophysiological mechanism that focuses, or "sharpens," outgoing motor commands from the M1 $(22,30)$. Indeed, where surround inhibition is reduced, high precision manual performance is often compromised [e.g., focal hand dystonia and Parkinson's disease $(22,31)]$. Furthermore, just as regulation of intracortical M1 inhibition is central to the fine tuning of complex action, it is also critical in those instances where the nervous system is required to terminate either a prepared or prepotent action (as per the Stop-signal task and the Go/No-go task). Case in point, intrahemispheric cortical inhibition within the M1 non-selectively increases (i.e., $\approx 160 \mathrm{~ms}$ ) before the successful, sudden cancelation of prepared or prepotent actions $(25,32,33)$, a process known as motor response inhibition (34).

Finally, during the performance of common unilateral movements, the nervous system has a natural tendency toward activating homologous muscles in the ipsilateral limb $(35,36)$. In these instances, interhemispheric cortical inhibition is required to prevent contralateral M1 activity traversing the corpus callosum to the ipsilateral M1, and the subsequent (unwanted) replication of action, or part thereof, in the ipsilateral hemisphere, known as "mirror movements" $(35,37,38)$. Indeed, reduced interhemispheric cortical inhibition has been reported in a number of patient groups where "mirror movements" are common (39-42).

In short, efficient and accurate motor control is predicated on the nervous system's capacity to regulate M1 cortical inhibitory mechanisms. Given this, it is perhaps unsurprising that there is a growing body of evidence suggesting that the integrity of $\mathrm{M} 1$ cortical inhibition may predict the quality of motor performance (43). For example, stronger intrahemispheric cortical inhibition regulation in the $\mathrm{M} 1$ at rest predicts better motor performance in neurotypical adults (44). Conversely, reduced intrahemispheric cortical inhibition within the M1 is associated with atypical motor function in patients with neurodevelopmental disorders [e.g., ADHD (45)], neurodegenerative 
diseases [e.g., Parkinson's disease $(46,47)$ and Huntington's disease $(48,49)]$ and neurological conditions where motor control is compromised [e.g., focal hand dystonia $(30,50,51)$ and Tourette's syndrome (52)].

\section{Transcranial Magnetic Stimulation (TMS): Insight Into Intrahemispheric and Interhemispheric Cortical Inhibition of the PMC}

Transcranial magnetic stimulation of the M1 has seen widespread use as a non-invasive means of investigating intra- and interhemispheric GABAergic activity $(53,54)$. A single-magnetic pulse delivered by TMS to the M1 in humans can produce activation in contralateral peripheral muscles. This muscle activation, also known as a motor-evoked potential (MEP), can be measured by electromyography (EMG) electrodes. With respect to intrahemispheric cortical inhibition, paired-pulse TMS (ppTMS) and cortical silent period (CSP) protocols are the most commonly adopted investigative methods $(55,56)$. ppTMS involves delivering two pulses to the same cortical point within the M1 in quick succession. The first pulse is a subthreshold "conditioning" pulse, which is then followed closely by a second suprathreshold "test" pulse. Depending on the time between the pulses, or "interstimulus interval" (ISI), a suppression of the muscle response is observed and is thought to reflect activity of GABAergic receptors within the M1 (57). Shorter ISIs (i.e., 2-5 ms) between pulses are thought to activate fast-acting $\mathrm{GABA}_{\mathrm{A}}$ receptors (58), a protocol referred to as short-interval cortical inhibition (SICI). Conversely, longer ISIs (i.e., $100-150 \mathrm{~ms}$ ), where both conditioning and test pulses are suprathreshold, are thought to activate relatively slower-acting $\mathrm{GABA}_{B}$ receptors (59), a protocol referred to as long-interval cortical inhibition (LICI). With respect to CSP, a single-TMS pulse is delivered to the cortical point on the M1 during voluntary muscle activation of the relevant peripheral muscle in the contralateral effector $(60,61)$. Generally, a period of suppressed muscle activity known as the "silent period" can be observed immediately following an initial TMS elicited MEP burst. CSPs are also thought to reflect GABA receptor activity (62); however, the nature of the receptor site is still a source of debate.

Finally, TMS procedures can also be reliably applied to index the integrity of interhemispheric cortical inhibition (63-65). While the latter can be inferred using various techniques, one of the more common is via the measurement of ipsilateral silent periods [ISPs $(64,66-68)$ ]. Here, a single-TMS pulse is delivered to the M1 on the ipsilateral side of an active muscle, while the contralateral homologous muscle is at rest. When an MEP is elicited in the contralateral hand as a result of the TMS pulse, a brief "silent period" in muscle activity can be observed in the homologous ipsilateral muscle. ISP is widely accepted as one of the TMS-evoked measures that reflect the brain's capacity to prevent transcallosal communication of motor commands (69-71), restricting neural activity to the contralateral M1 during unilateral movements and preventing unwanted mirror movements (35). Like measures of intrahemispheric cortical inhibition, interhemispheric cortical inhibition is also thought to be dependent on both $\mathrm{GABA}_{\mathrm{A}}$ and $\mathrm{GABA}_{\mathrm{B}}$ receptor activity (72).

\section{M1 Cortical Inhibition and DCD}

As outlined earlier, there is compelling evidence indicating that the efficiency with which M1 cortical inhibition is regulated predicts motor ability [e.g., Ref. (44)]. Indeed, reduced cortical inhibition within the M1 predicts compromised motor function in neurodevelopmental disorders that commonly co-occur with DCD, such as ADHD (45). Despite this, no study to our knowledge has directly investigated the integrity of cortical inhibitory processes in DCD, a disorder primarily characterized by impaired motor control.

There are, however, several converging lines of evidence that support the view that cortical inhibition of the M1 may be compromised in DCD. Recent experimental work is replete with evidence that individuals with DCD present with behavioral phenotypes that are traditionally associated with reduced intra- and interhemispheric cortical inhibition. For example, a number of studies have shown that individuals with DCD demonstrate an increased incidence of the type of "mirror movements" symptomatic of poor interhemispheric inhibition (73-77). Likewise, children with DCD have consistently shown difficulties performing tasks that require the sudden cancelation of both prepared or prepotent movements (78-87). Since motor response inhibition is dependent on efficient intrahemispheric cortical inhibition within the M1 $(25,33)$, poor performance could feasibly occur due to poor modulation of intrahemispheric GABAergic activity. It should also be noted that individuals with atypical motor skills often show poor performance on various neuropsychological tests of inhibition (88). However, it is often difficult to discern whether such deficits are motoric or cognitive in nature given the cognitive demands of these tasks (89), the latter of which are unlikely to be rooted in the M1. Finally, while the inefficient, slow, and highly variable performance during complex manual tasks typical of individuals with DCD is unlikely to have a single-neurological correlate, it is nonetheless consistent with reduced surround inhibition. Again, surround inhibition is a process dependent on selective upregulation and deregulation of cortical inhibition within the M1 for the purpose of "fine tuning" motor commands (22). Taken together, we argue that the clinical and experimental profile of individuals with DCD is consistent with pathophysiology of GABAergic activity within the M1, which warrants investigation.

\section{The Present Paper}

The aim of this study was to investigate the integrity of intra- and interhemispheric cortical inhibition within the M1 in a sample of young adults with DCD using TMS. Similarly to recent work, given the heterogeneity associated with samples of individuals with DCD, we opted to include young adults with DCD rather than children to control for the subgroup of children who "out-grow" the condition thereby reducing one common source of variability $(90,91)$. Given the exploratory nature of this study, M1 cortical inhibition was measured using a battery of single-pulse TMS and ppTMS protocols commonly adopted to investigate intra- and interhemispheric cortical inhibition. Based on evidence that 
cortical inhibition within the M1 predicts motor function in neurotypical and atypical populations, and evidence that individuals with DCD regularly present with behavioral markers consistent with atypical regulation of GABAergic mechanisms in the M1, it was predicted that individuals with DCD would present with reduced intra- and interhemispheric cortical inhibition relative to controls.

\section{MATERIALS AND METHODS}

\section{Participants}

The sample comprised 8 adults with DCD ( 4 males and 4 females, $\mathrm{M}_{\mathrm{age}}=23.75, \mathrm{SD}=1.67$, age range $=21-32$ ) and 10 typically developing (TD) controls (6 males and 4 females, $M_{\text {age }}=26.00$, $\mathrm{SD}=4.24$, age range $=21-26)$. All participants self-reported being right handed, gave written informed consent, and were screened to ensure they were free of TMS contraindications. No participants reported taking medication that would contraindicate TMS, and none reported experiencing any negative side-effects during or following TMS. The project received ethical clearance from Deakin University Human Research Ethics Committee.

Participants were recruited through advertisements placed on university websites at an Australian University and social media outlets (i.e., Facebook), targeting both individuals with motor difficulties and typical motor skill. All participants were screened using methods that have been successful by ours and other research groups in identifying adults with DCD (90-94). Prospective participants first completed the Adult Dyspraxia/ Developmental Coordination Checklist [ADC (95)], and those who were deemed eligible then had their motor ability assessed using the Bruininks-Oseretsky Test of Motor Proficiency, Second Edition [BOT-2 (96)]. The BOT-2 is a well-validated standardized measure of motor skill, containing subtests that index each participant's "Fine manual control," "Manual coordination," "Body coordination," and "Strength and agility." Based on each participant's performance on each of the subtests, "Total Motor Composite" scores $(M=50, S D=10)$ are then generated for each participant, providing an index of their age-normed motor ability. The BOT-2 was adopted over other standardized measures of motor ability based on recent reviews, which found the BOT-2 to be the most valid and reliable battery available for identifying motor difficulties in young Australian adults $(97,98)$.

All participants with DCD were selected according to DSM-5 criteria and in accordance with recent guidelines for identifying DCD in adults (99). Participants were considered to have DCD if their motor proficiency was significantly below that expected given their age (Criterion A), as indicated by BOT-2 "Total Motor Composite" scores at or below the 15th percentile (14). In total, 13 participants met this criterion. These motor difficulties must have significantly impacted their ability to undertake daily activities involving movement (Criterion B) and arose in childhood (Criterion C), as determined using the ADC. The ADC is a 40 -item questionnaire designed to identify current and childhood difficulties with completing daily living tasks related to motor function. The $\mathrm{ADC}$ is often used to determine whether poor motor skill is associated with a reduced ability to perform tasks of everyday living (93, 100-102). While ideal for addressing Criteria B and $\mathrm{C}$, conjecture remains surrounding appropriate cutoffs. Based on a recent study which had developed $95 \%$ confidence intervals for total $\left(\mathrm{CI}_{95 \%}: 21.26_{\text {Mean }} \pm 3.27\right)$ and child $\left(\mathrm{CI}_{95 \%}: 4.26\right.$ Mean \pm 0.86$)$ ADC scores using 47 healthy young Australian adults (93), participants who had met Criterion A whom also scored above the $95 \%$ confidence interval cutoff for the total (i.e., 25 or above) and child scores (i.e., 6 and above) were deemed to have met criteria $B$ and $C$ respectively. Twelve of the 13 participants who met Criterion A also met Criteria $\mathrm{B}$ and $\mathrm{C}$. None of these participants reported a previous diagnosis of any neurological or medical condition affecting movement (e.g., cerebral palsy) and were deemed to have had intelligence at least in the normal range since they were recruited through the University setting and/or had completed an undergraduate degree (Criterion D). Finally, while 12 participants met the above criteria for DCD, only 8 met the required medical criteria to undergo TMS. Accordingly, our final sample of participants with DCD consisted of $(n) 8$. While no participant had a formal diagnosis of DCD, we can be confident that participants in our DCD group met criteria as outlined earlier. Using the same screening procedure, all controls were confirmed to have ageappropriate motor abilities, as indicated by a percentile ranking above the 20th percentile for "Total Motor Composite" scores of the BOT-2 [see also Ref. $(83,93,100)$ ] and were free of any self-reported medical or neurological impairments.

\section{Transcranial Magnetic Stimulation}

Single-pulse TMS (Magstim-200 stimulator, Magstim Company Ltd., UK) was administered to M1 using a hand-held, $70 \mathrm{~mm}$ figure-of-eight coil that was positioned against the scalp using the orthodox method (handle pointing backwards and angled $45^{\circ}$ away from midline). ppTMS was administered using the same coil and two-Magstim-200 2 stimulators that were combined via a BiStim ${ }^{2}$ module. MEPs were recorded from the right-hand first dorsal interosseous (FDI) muscle using three EMG self-adhesive electrodes: an active electrode placed over the muscle belly of the FDI, a reference electrode over the interphalangeal joint of the right index finger and a ground electrode on the ulnar styloid process. For measurements of ISP, EMG was recorded from the FDI of the left hand, ipsilateral to the coil using LabChart v7 (66). EMG was acquired via a PowerLab/4SP system (AD Instruments, Colorado Springs, CO, USA). Grip force was measured via an AD instruments MLT004/ST grip force transducer.

Single-pulse TMS was used to locate the site of the M1 on the left hemisphere that would produce a maximal response in the right FDI. This "hotspot" location was marked and used as the site for TMS delivery for the rest of the session. Resting-motor threshold (RMT) was defined as the minimum intensity that produced a peak-to-peak MEP of $>50 \mu \mathrm{V}$ in at least 5 out of 10 consecutive trials (103). Active-motor threshold (AMT) was defined as the lowest stimulation intensity that, during tonic muscle contraction ( $10 \%$ of maximal contraction as assessed via a grip force transducer), produced a peak-to-peak MEP of $>200 \mu \mathrm{V}$ in at least 5 out of 10 consecutive trials (104). 


\section{Intrahemispheric Cortical Inhibition}

Twenty baseline MEPs were then recorded following singlepulse TMS delivered at 4 -s intervals, at 120\% RMT. Cortical inhibition was then assessed via a number of ppTMS paradigms. Short-interval cortical inhibition (SICI) was recorded following the delivery of a subthreshold conditioning pulse (90\% AMT), followed $2 \mathrm{~ms}$ later by a suprathreshold test pulse [120\% RMT $(105,106)]$. LICI was then recorded following the delivery of two suprathreshold pulses (120\% RMT), separated by $100 \mathrm{~ms}$ (107-109). Both SICI and LICI were delivered at 4-s intervals. Ten recordings were taken for SICI and LICI, respectively. CSP was then recorded following single-pulse TMS (130\% AMT) at 4-s inter-trial intervals (ITI) while participants maintained voluntary muscle contraction at $\sim 20 \%$ of maximal voluntary contraction (MVC), as measured by the grip force transducer $(61,110)$. Fifteen recordings were taken for CSP.

\section{Interhemispheric Cortical Inhibition}

Ipsilateral silent period and related EMG activity were recorded from the FDI of the left hand, following the delivery of 15 TMS pulses (150\% RMT) to the ipsilateral hemisphere (111). Approximately $3 \mathrm{~s}$ before each pulse, participants were instructed to perform $100 \%$ of MVC of their left hand, by applying force with their index finger to the force transducer. The pulses were delivered with an ITI of $10 \mathrm{~s}$, during which participants were instructed to relax their hand to prevent fatigue (112).

\section{Data Preparation}

Motor-evoked potentials were analyzed by determining their peak-to-peak millivolt amplitudes. For SICI, each participant's median conditioned peak-to-peak MEP was divided by their respective median unconditioned peak-to-peak MEP measured at baseline. The resulting value was then multiplied by 100 and subtracted from 100 to represent the percentage of inhibition of the test pulse [SICI (\%)]

$$
\operatorname{SICI}(\%)=100-\left[\left(\frac{C}{\mathrm{NC}}\right) \times 100\right] \text {. }
$$

For LICI, each participant's median peak-to-peak MEP in response to the second TMS pulse (the conditioned pulse) was divided by their median peak-to-peak MEP in response to the first TMS pulse (the non-conditioned pulse). The resulting value was then multiplied by 100 and subtracted from 100 to represent the percentage of inhibition of the second suprathreshold test pulse [LICI (\%)]

$$
\operatorname{LICI}(\%)=100-\left[\left(\frac{C}{\mathrm{NC}}\right) \times 100\right] .
$$

For CSP, the onset and offset of the silent period was determined using the objective graphical method described by Garvey et al. (113). The CSP duration ( $\left.\mathrm{CSP}_{\text {duration }}\right)$ was defined as the mean of the temporal difference (in $\mathrm{ms}$ ) between $\mathrm{CSP}_{\text {onset }}$ and $\mathrm{CSP}_{\text {offset }}\left(\mathrm{CSP}_{\text {duration }}=\mathrm{CSP}_{\text {offset }}-\mathrm{CSP}_{\text {onset }}\right)$. Furthermore, since the duration of silent periods depend on stimulus intensities (61), the $\mathrm{CSP}_{\text {duration }}$ of each trial was divided by the peak-to-peak
MEP amplitude of the same trial ( $\left.\mathrm{CSP}_{\text {ratio }}\right)$ to reduce intersubject variability. The mean $\mathrm{CSP}_{\text {ratio }}$ was then calculated and used for subsequent analyses. Although not presented here, we note that the reported profile of between group comparisons for CSP did not differ appreciably without this normalization

$$
\mathrm{CSP}_{\text {ratio }}=\frac{\mathrm{CSP}_{\text {durationin ms }}}{\text { peak }- \text { to }- \text { peak MEP amplitude }} \text {. }
$$

The graphical method described earlier was also used to determine onset and offset for ISPs (113). The duration of ISP $\left(\mathrm{ISP}_{\text {duration }}\right)$ was defined as the mean temporal difference between $I_{S P}$ onset and $I_{S P}$ offset $\left(I_{S P}\right.$ duration $\left.=I S P_{\text {offset }}-I_{S P} P_{\text {onset }}\right)$. Given that the duration of ISPs also seems to be affected by stimulus intensities $(61,114)$, the ISP $_{\text {duration }}$ of each trial was also divided by the peakto-peak MEP amplitude evoked in the contralateral FDI of the same trial $\left(\right.$ ISP $\left._{\text {ratio }}\right)$. The mean ISP $_{\text {ratio }}$ was then calculated and used for subsequent analyses. Although not presented here, we note that the reported profile of between group comparisons for ISP did not differ appreciably without this normalization

$$
\mathrm{ISP}_{\text {ratio }}=\frac{\mathrm{ISP}_{\text {duration in ms }}}{\text { peak }- \text { to }- \text { peak MEP amplitude of contralateral FDI }} \text {. }
$$

\section{Data Analysis}

Group comparisons of intra- and interhemispheric cortical inhibition were conducted using Independent Samples $t$-tests for outcome measures where assumptions of normality were met (i.e., $\mathrm{CSP}_{\text {ratio }}$ and $\mathrm{ISP}_{\text {ratio }}$ ). Where assumptions were violated, Mann-Whitney $U$-tests were adopted [i.e., SICI (\%) and LICI (\%)]. Where significant group differences were observed, Pearson's correlation analyses were conducted between those cortical inhibition measures and scale scores $(M=15, S D=5)$ of relevant subtests from the BOT-2 requiring the use of the FDI. Since group differences were only observed on measures of interhemispheric cortical inhibition (see below), the purpose of the subsequent analysis was to determine whether this effect in DCD was associated with motor performance deficits. Only those subtests (i.e., "Manual Dexterity") that placed greater demands on interhemispheric cortical inhibition (i.e., bimanual coordination of left and right hands) were subjected to correlations with our TMS measure of interhemispheric cortical inhibition.

\section{RESULTS}

All participants completed all measures. No group differences were observed for RMT or AMT. Baseline MEPs were also comparable between groups. As RMTs, AMTs and baseline MEPs were comparable between groups, and stimulator output percentages for SICI, LICI, CSP, and ISP are all based on RMT and AMT percentages, comparisons on stimulus intensities for SICI, LICI, CSP, and ISP were deemed redundant and were not conducted. See Table 1 below.

\section{Intrahemispheric Cortical Inhibition}

Comparisons of mean intrahemispheric cortical inhibition [SICI (\%), LICI (\%), and $\mathrm{CSP}_{\text {ratio }}$ ] found no significant differences between groups (see Table 2). Scatterplots for the 
TABLE 1 | Mean and SDs (in parentheses) of RMT and AMT and median baseline MEPs for DCD and TD groups.

\begin{tabular}{lcccccc}
\hline & DCD & TD & $\boldsymbol{t}$ & df & $\boldsymbol{p}$ & $\begin{array}{c}\text { Effect } \\
\text { size }\left(\boldsymbol{\eta}^{2}\right)\end{array}$ \\
\hline RMT (\%) & $46.50(9.17)$ & $48.13(6.85)$ & 0.42 & 16 & 0.683 & 0.011 \\
AMT (\%) & $38.10(8.39)$ & $39.38(6.37)$ & 0.68 & 16 & 0.727 & 0.008 \\
Baseline & $1.10(0.54)$ & $1.27(0.74)$ & 0.18 & 16 & 0.858 & 0.002 \\
MEPs (mV) & & & & & & \\
\hline
\end{tabular}

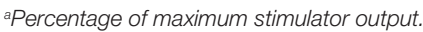

RMT, resting-motor threshold; AMT, active-motor threshold; DCD, developmental coordination disorder; TD, typically developing; MEPS, motor-evoked potentials.

TABLE 2 | Outcome measures for intrahemispheric cortical inhibition.

\begin{tabular}{llllllll}
\hline & DCD & TD & Statistic & df & $p$ & $\begin{array}{l}\text { Effect } \\
\text { size }\end{array}$ \\
\hline $\mathrm{SICl}(\%)$ & $54.80^{\text {Median }}$ & $73.51^{\text {Median }}$ & $30.00^{\mathrm{U}}$ & - & 0.329 & $0.949^{z}$ \\
$\mathrm{LICl}(\%)$ & $79.98^{\text {Median }}$ & $88.57^{\text {Median }}$ & $36.00^{\mathrm{U}}$ & - & 0.648 & $0.738^{z}$ \\
$\mathrm{CSP}_{\text {ratio }}$ & $13.61^{\text {Mean }}(7.41)$ & $17.67^{\text {Mean }}(9.68)$ & $0.978^{\mathrm{t}}$ & 16 & 0.343 & $0.056^{\eta 2}$ \\
\hline
\end{tabular}

Mean Group mean and SD in parentheses.

Median Group median.

uCompared using a Mann-Whitney U-test.

${ }^{t}$ Compared using an Independent Samples t-test.

zKolmogorov-Smirnov Z.

$\eta^{2}$ Eta squared.

$D C D$, developmental coordination disorder; TD, typically developing; SICI, short-interval cortical inhibition; LICI, long-interval cortical inhibition; CSP, cortical silent period.

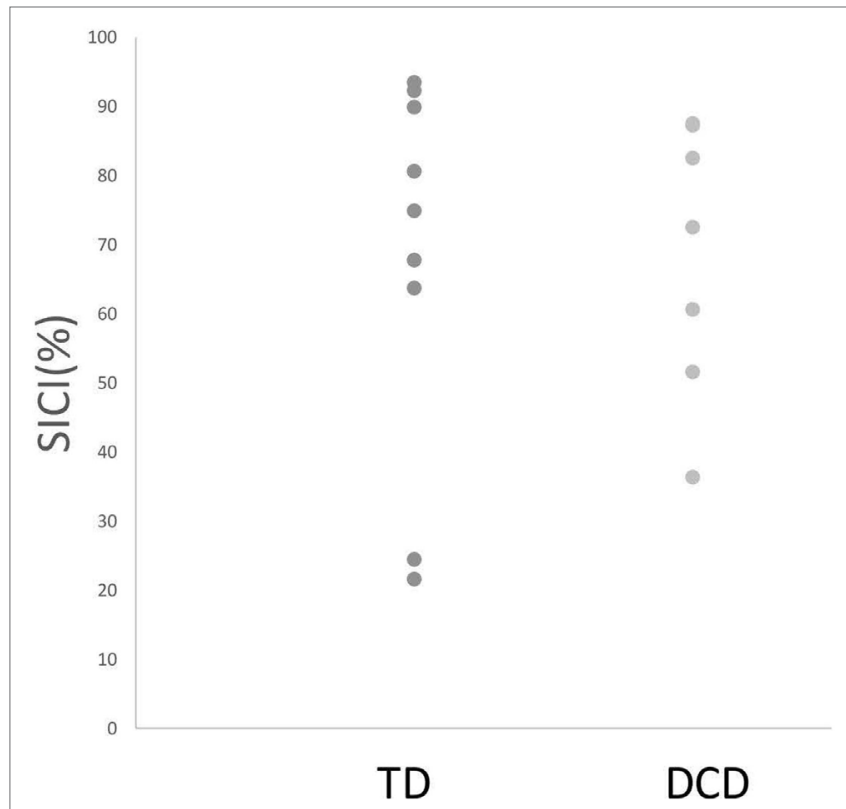

FIGURE 1 | Median SICI (\%) for both TD and DCD groups. Note: Due to the scale, one TD and one DCD participants are not shown in this figure because they demonstrated facilitation (and hence SICI \% fell below 0). Abbreviations: $\mathrm{SICl}$, short-interval cortical inhibition; TD, typically developing; DCD, developmental coordination disorder.

intrahemispheric cortical inhibition measures are presented for DCD and TD groups in Figures 1-3 for SICI (\%), LICI (\%), and $\mathrm{CSP}_{\text {ratio }}$ respectively.
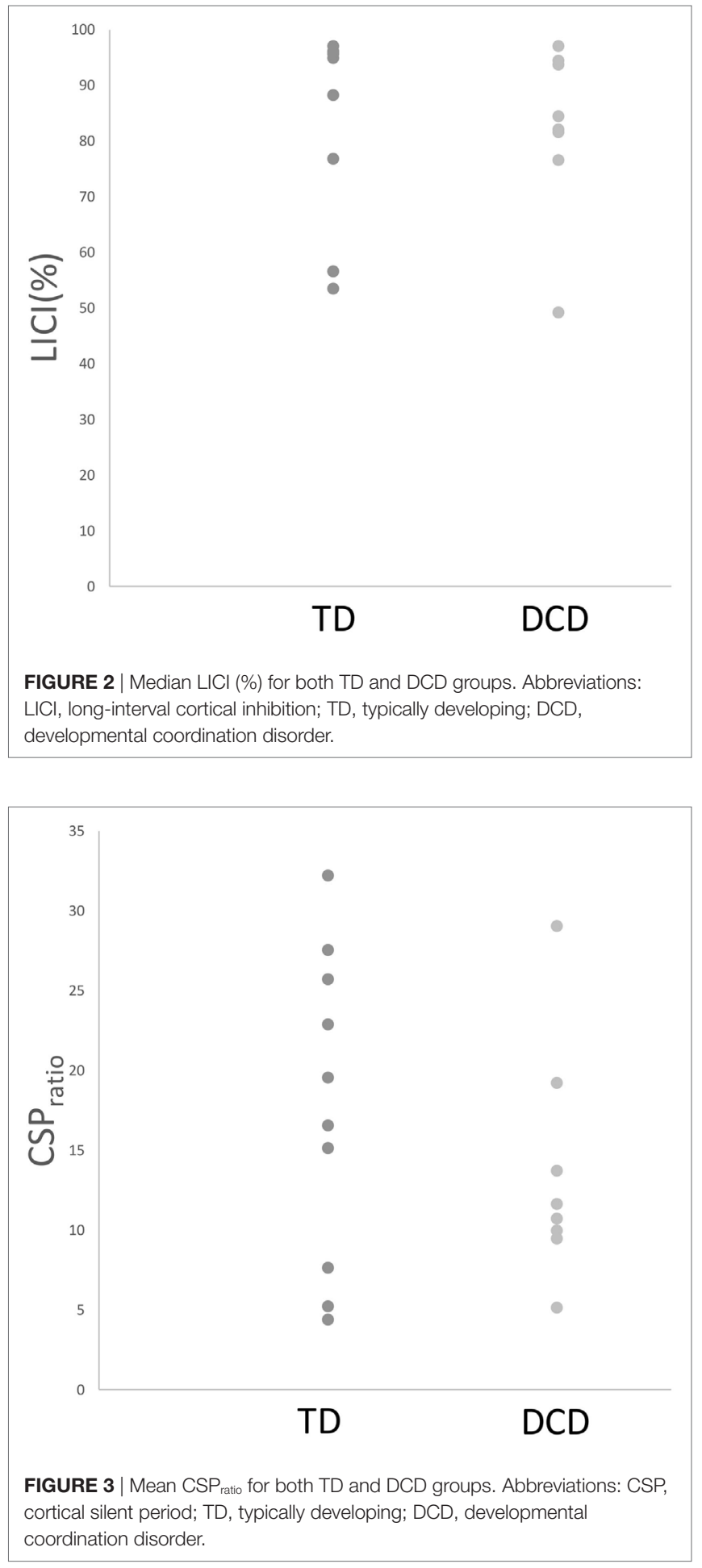

\section{Interhemispheric Cortical Inhibition}

In regards to interhemispheric cortical inhibition, mean ISP $_{\text {ratios }}$ were significantly smaller in the DCD group $\mathrm{CI}_{95 \%}: 3.81_{\text {Mean }} \pm 0.61$ relative to TD controls $\mathrm{CI}_{95 \%}$ : $6.08_{\text {Mean }} \pm 1.32$ (see Table 3 ). Individual

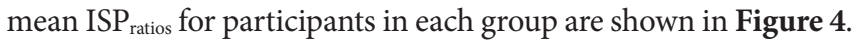
As can be observed, seven of eight DCD participants (or 88\%) fell below the lower-bound $\mathrm{CI}_{95 \%}$ threshold of the TD group (i.e., 4.76). 


\section{Correlation Between ISP ratios and BOT-2 Subtests}

Pearson's correlation revealed a significant, medium to strong, positive correlation between mean ISP $_{\text {ratios }}$ and "Manual

TABLE 3 | Mean and SD (in parentheses) of outcome measure for interhemispheric cortical inhibition.

\begin{tabular}{ccccccc}
\hline DCD & TD & $\boldsymbol{t}$ & df & $\boldsymbol{p}$ & Effect size $\left(\boldsymbol{\eta}^{2}\right)$ \\
\hline ISP $_{\text {ratio }}$ & $3.81(0.73)$ & $6.08(1.84)$ & $3.56^{\mathrm{a}}$ & 12.29 & 0.004 & 0.401
\end{tabular}

aViolation of assumption of homogeneous variance was present, equal variances not assumed.

$D C D$, developmental coordination disorder; TD, typically developing; ISP, ipsilateral silent period.

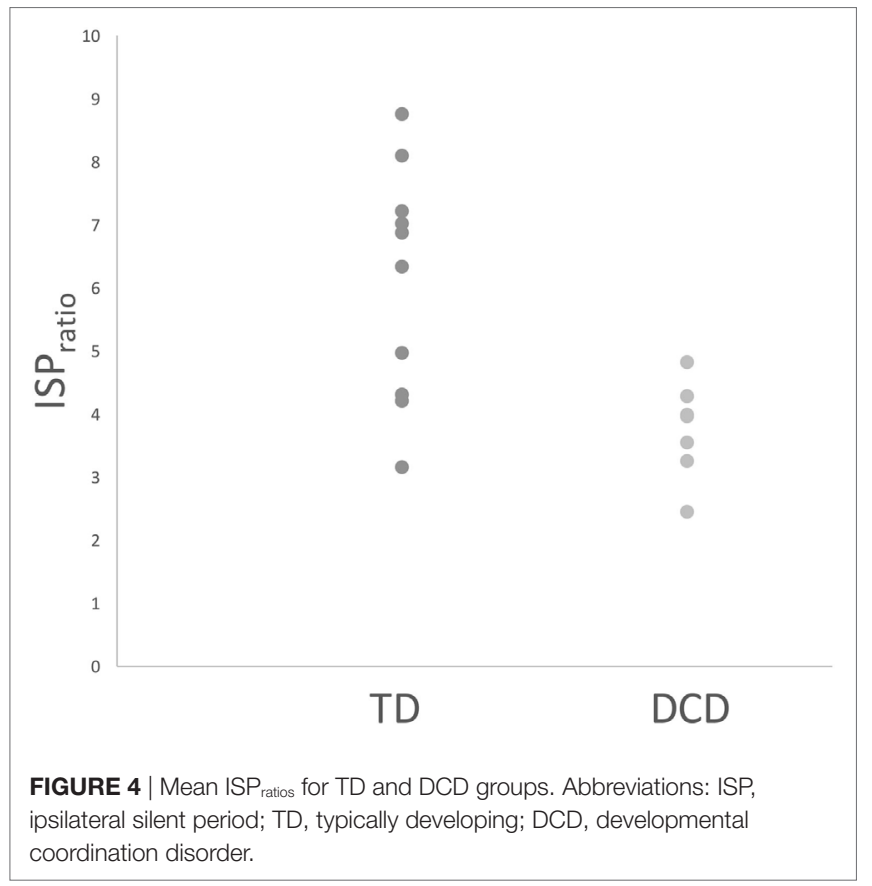

Dexterity" scale scores of the BOT-2, $r=0.48, n=18$, and $p=0.044$. A scatterplot illustrating this correlation is presented below in Figure 5.

\section{DISCUSSION}

The aim of this study was to investigate the integrity of intraand interhemispheric cortical inhibition of the M1 in adults with DCD using a battery of single-pulse TMS and ppTMS protocols that are commonly implemented to measure these respective facets of cortical inhibition. Contrary to expectations, our results suggested that intrahemispheric cortical inhibition at rest is preserved in DCD, with no group differences observed on

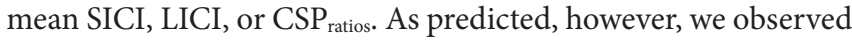
significant differences in mean ISP $_{\text {ratios }}$ between groups, a commonly adopted measure of interhemispheric cortical inhibition. Indeed, adults with DCD showed significantly smaller mean ISP $_{\text {ratios }}$ in comparison to healthy controls. Interestingly, the reduced interhemispheric cortical inhibition in the DCD group was also associated with poorer performance on subtests of the BOT-2 requiring bimanual coordination, shown by a significant correlation between mean ISP $\mathrm{I}_{\text {ratios }}$ and performance on the BOT-2 manual dexterity subtest across groups.

\section{Intrahemispheric Cortical Inhibition Is Preserved in DCD}

Based on behavioral findings indicating that individuals with DCD often display phenotypes consistent with reduced intrahemispheric cortical inhibition within the M1 (73-80, 84-87), we hypothesized that intrahemispheric cortical inhibition may be reduced in DCD. Contrary to expectations, comparisons between DCD and TD groups on a broad range of gold-standard indices of intrahemispheric cortical inhibition [i.e., SICI (\%), LICI (\%), and $\mathrm{CSP}_{\text {ratio }}$ ] failed to reveal statistically meaningful differences. This was particularly surprising given the behavioral phenotypes described earlier had been previously reported to rely on intrahemispheric inhibitory mechanisms within the

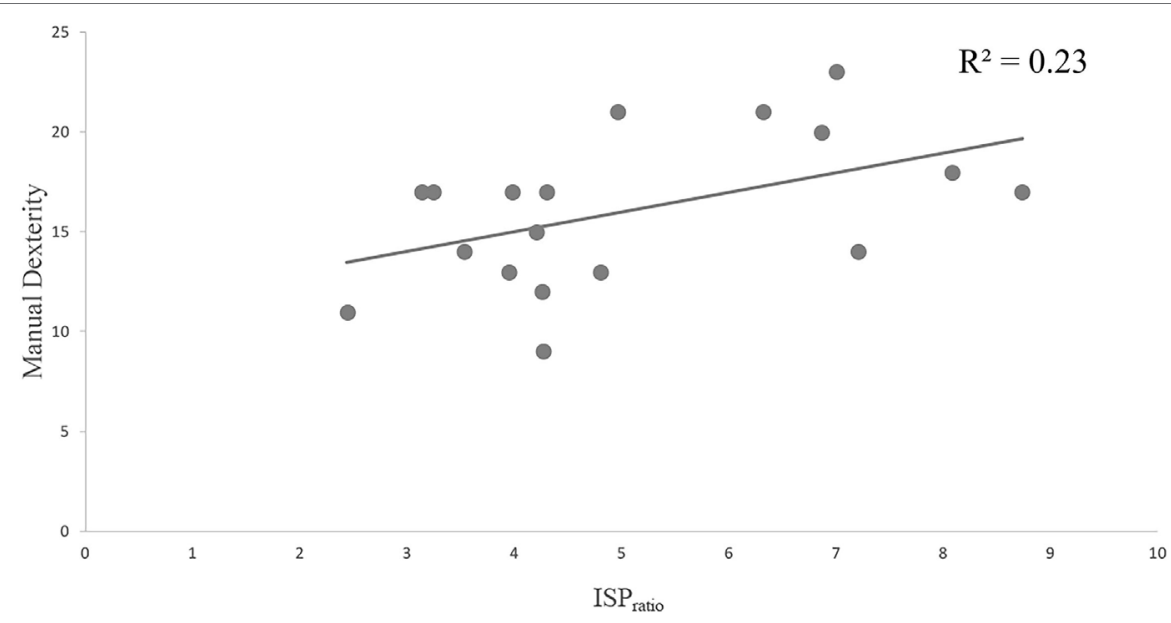

FIGURE 5 | Pearson's correlation between performance on the manual dexterity subtest from the BOT-2 and mean ISP ratios. Abbreviations: BOT-2, BruininksOseretsky Test of Motor Proficiency, Second Edition; ISP, ipsilateral silent period. 
M1 $(25,33)$. While it is possible that our modest sample size may have contributed to this lack of effect, we argue that the very small effect size (see Table 2) and considerable overlap in within-group variability across groups with and without DCD (see Figures 1-3) indicate that low study power is unlikely to account for our null findings. Thus, our results indicate that intrahemispheric cortical inhibition may be preserved in DCD, and, hence, is unlikely to be associated with previous reports of a decreased capacity to engage motor inhibition in this group as previously argued.

It is important to note that two of our measures of intrahemispheric cortical inhibition [i.e., SICI (\%) and LICI (\%)] were taken with the participant at rest (i.e., not performing an action) while the other (i.e., CSP) was taken while participants performed a very simple motor task (i.e., abduction of the FDI). Differences in resting-state cortical inhibition such as those adopted here have previously been detected between healthy controls and individuals with neurodevelopmental disorders where motor function is often impaired, such as ADHD (45). Furthermore, resting-state measures of intrahemispheric cortical inhibition have shown to predict motor competence in healthy individuals (44). Thus, we can be confident that measures of within hemisphere M1 cortical inhibition taken when the motor system is largely inactive (or at least not conducting complex movement) clearly provide important insight into the GABAergic properties of the motor system $(44,45,115)$. These, it would seem, are preserved in individuals with DCD. However, resting-state measures such as those adopted here may not necessarily provide insight into how cortical inhibition within the M1 is modulated during fluent purposive action. As noted previously, successful inhibition of a prepared or prepotent action is typically preceded by an increase in cortical inhibition [as measured using SICI $(25,33)$, supporting the view that modulation of cortical inhibition on the fly is central to the cancelation of movement [i.e., motor inhibition $(19,116)]$. Thus, while the data from this study do suggest that the GABAergic processes within the inactive contralateral M1 may be preserved in DCD, it remains to be seen whether this group is able to modulate these processes flexibly during movement to support the suppression of unwanted movement, as is observed in healthy adults. Behaviorally, recent work has shown that children with DCD are less able to integrate inhibitory control with movements "online" to support corrections to movement mid-flight following unexpected perturbations to the reach target. Indeed, during double-step reaching tasks when the target of movements moves laterally following movement onset, children with DCD are more likely to complete movements toward a prepotent yet incorrect target before re-directing their action toward the new target [e.g., Ref. (78)], although reports of the effect are not constant [e.g., Ref. (84)]. Since inhibition of such movements requires active modulation of intracortical M1 inhibition (25), it is possible that this behavioral profile may be subserved by a decreased ability to modulate GABAergic systems flexibly during active movement. This remains speculative, however, and should be the focus of future work. Still, taken together, our data suggest that intrahemispheric cortical inhibition is preserved in DCD, at least when the motor system is not engaged in complex movement.

\section{Interhemispheric Cortical Inhibition Is Reduced in DCD}

Comparison of ISP $_{\text {ratios }}$ between DCD and TD groups found that the DCD group had significantly smaller ISP ratios when compared with their TD counterparts. As discussed, ISPs are commonly used as a metric for indexing cortical inhibitory mechanisms involved in the suppression of unwanted transcallosal communication of motor commands across M1 hemispheres during lateralized movement $(64,66,68,117)$. It is a process thought to be particularly important for the accurate and efficient production of strict unimanual movements, and bimanual coordination where inhibition of homologous ipsilateral muscles is critical $(35,66)$. Thus, our finding that individuals with DCD had reduced ISP $_{\text {ratios }}$ relative to controls supports the hypothesis that interhemispheric cortical inhibition of M1 activity may be atypical in individuals with DCD. Importantly, assessment of within-group differences showed that all but one participant in the DCD group (88\%) fell below the $95 \%$ CI of the control group on mean ISP ratios. $_{\text {Thus, not only }}$ do those with DCD demonstrate decreased interhemispheric inhibition of M1 activity at a group level, but the effect appears to hold for a substantial proportion of individuals in this group. Furthermore, we observed that smaller ISP $_{\text {ratios }}$ were associated with decreased performance on a subtest of the BOT-2 requiring bimanual coordination, and where the FDI was also used as the main effector (i.e., "Manual Dexterity"), with a compelling $30 \%$ of the variability in performance being explained by mean ISP $_{\text {ratios }}$ (see Figure 5). These data are not only consistent with earlier accounts that interhemispheric inhibition of M1 activity is critical for accurate and efficient bimanual movements (118) but demonstrate that reduced interhemispheric M1 inhibition in DCD may be associated with their poor motor function (at least with respect to hand movement).

Furthermore, the reduced interhemispheric cortical inhibition identified in our DCD group is consistent with the growing number of behavioral studies which have consistently found more unwanted activity of ipsilateral homologous muscles (i.e., mirror movements) during lateralized unimanual motor tasks $(76,77)$ and bimanual coordination tasks $(73,74,119)$ in DCD than TD controls. For example, during a recent finger tapping task which requires children to switch from bimanual to unimanual finger tapping, children with DCD made more additional taps of a non-cued finger when required to switch from tapping with both fingers to tapping with just one (119). Similarly, in a recent fMRI study, children with DCD displayed more unwanted activation of a non-cued hand during finger sequencing or fist clenching of the contralateral hand than their TD peers (77). However, despite the presence of such mirror movements, the authors of this particular fMRI study were unable to identify any activation deficits that could explain the presence of these unwanted actions. Interestingly a recent EEG study found that the increased incidence of mirror movements in DCD may be associated with reduced interhemispheric communication of inhibitory information (73). Specifically, children with DCD made more mirror movements during performance of a novel bimanual coordination task than their TD peers, with increased mirror movements correlating with lower cortico-cortical 
coherence between frontocentral regions of both hemispheres. Our neurophysiological findings are also in line with recent structural MRI work showing functional anisotropy reductions in calossal regions in children with DCD, the major communication pathway between hemispheres (120). While speculative, the decreased interhemispheric cortical inhibition of M1 activity observed here in DCD could certainly be a plausible mechanism with which to explain the reduced interhemispheric communication in individuals with DCD (73) and the greater mirror movements observed.

While the results of the present are promising, we acknowledge that we can only generalize these findings to the dominant M1 (left in this case), since cortical inhibition of the non-dominant hemisphere was not evaluated. That is, while our study shows that interhemispheric inhibition of right-hemispheric activity from the dominant left hemisphere is reduced in DCD, it cannot be assumed that this is also the case for the opposite hemisphere. Indeed, asymmetry in interhemispheric cortical inhibition has been noted in both patient groups with reduced ISPs (41) and controls (121). This issue remains an important avenue for future work, particularly given that excitability and inhibition within the M1 has previously been shown to be amenable to therapy $(122,123)$. Furthermore, as noted, by definition adults with DCD represent as a group of individuals for whom childhood motor difficulties persisted into adulthood. Thus, we must be mindful of generalizing the findings of our study to children with DCD not just because of the more obvious developmental factors, but also since any sample of children with DCD is likely to contain a subgroup of individuals whose motor difficulties dissipate with age and who thus represent a different subpopulation of individuals with motor difficulties to the one of interest here.

\section{CONCLUSION}

This study was the first of its kind to examine the integrity of intra- and interhemispheric cortical inhibition in DCD using single-pulse TMS and ppTMS paradigms. While intrahemispheric

\section{REFERENCES}

1. American Psychiatric Association. Diagnostic and statistical manual of mental disorders (DSM-5 $\left.{ }^{\circledR}\right)$. American Psychiatric Pub (2013).

2. Rivilis I, Hay J, Cairney J, Klentrou P, Liu J, Faught BE. Physical activity and fitness in children with developmental coordination disorder: a systematic review. Res Dev Disabil (2011) 32(3):894-910. doi:10.1016/j.ridd.2011.01.017

3. Summers J, Larkin D, Dewey D. Activities of daily living in children with developmental coordination disorder: dressing, personal hygiene, and eating skills. Hum Mov Sci (2008) 27(2):215-29. doi:10.1016/j.humov.2008.02.002

4. Lingam R, Hunt L, Golding J, Jongmans M, Emond A. Prevalence of developmental coordination disorder using the DSM-IV at 7 years of age: a UK population-based study. Pediatrics (2009) 123(4):e693-700. doi:10.1542/ peds.2008-1770

5. Biotteau M, Chaix Y, Blais M, Tallet J, Péran P, Albaret J-M. Neural signature of DCD: a critical review of MRI neuroimaging studies. Front Neurol (2016) 7:227. doi:10.3389/fneur.2016.00227

6. Cantell MH, Smyth MM, Ahonen TP. Two distinct pathways for developmental coordination disorder: persistence and resolution. Hum Mov Sci (2003) 22(4-5):413-31. doi:10.1016/j.humov.2003.09.002

7. Cousins M, Smyth MM. Developmental coordination impairments in adulthood. Hum Mov Sci (2003) 22(4):433-59. doi:10.1016/j.humov.2003.09.003 cortical inhibition was comparable between DCD and TD groups, interhemispheric cortical inhibition was reduced in DCD. An additional correlation analysis conducted between ISP $_{\text {ratios }}$ and the "Manual Dexterity" subtest of the BOT-2 found that TMS evoked ISPs were significantly related to performance on subtests requiring fine bimanual coordination. Taken together, our findings suggest that not only do individuals with DCD demonstrate reduced interhemispheric cortical inhibition of M1 activity, but that this may predict reduced motor performance in this group. Directions for future work and clinical implications were discussed. Our data are partly consistent with the view that cortical inhibition of M1 activity may be compromised in individuals with DCD and are consistent with behavioral accounts of reduced motor inhibition in this group.

\section{ETHICS STATEMENT}

This study was carried out in accordance with the recommendations of Deakin University Human Research Ethics Committee (DUHREC) with informed consent from all subjects. All subjects gave written informed consent in accordance with the Declaration of Helsinki. The protocol was approved by DUHREC.

\section{AUTHOR CONTRIBUTIONS}

$\mathrm{JH}, \mathrm{CH}$, and IF contributed to all stages of this study, including project conception and design, data collection, analysis, and final write-up of the manuscript. PE and W-pT contributed substantially to project conceptualization and manuscript preparation. $\mathrm{PB}$ contributed to data collection and manuscript preparation.

\section{ACKNOWLEDGMENTS}

The authors would like to show our gratitude to all the participants involved in this study. They would also like to thank Michael Do and Brendan Major for their input and assistance on the development of the TMS protocol used in this study.

8. Kirby A, Sugden D, Beveridge S, Edwards L. Developmental co-ordination disorder (DCD) in adolescents and adults in further and higher education. J Res Spec Edu Needs (2008) 8(3):120-31. doi:10.1111/j.1471-3802.2008.00111.x

9. Purcell C, Scott-Roberts S, Kirby A. Implications of DSM-5 for recognising adults with developmental coordination disorder (DCD). Br J Occup Ther (2015) 78(5):295-302. doi:10.1177/0308022614565113

10. Hill EL, Brown D. Mood impairments in adults previously diagnosed with developmental coordination disorder. J Ment Health (2013) 22(4):334-40. d oi:10.3109/09638237.2012.745187

11. Kirby A, Williams N, Thomas M, Hill EL. Self-reported mood, general health, wellbeing and employment status in adults with suspected DCD. Res Dev Disabil (2013) 34(4):1357-64. doi:10.1016/j.ridd.2013.01.003

12. Gomez A, Sirigu A. Developmental coordination disorder: core sensorimotor deficits, neurobiology and etiology. Neuropsychologia (2015) 79:272-87. doi:10.1016/j.neuropsychologia.2015.09.032

13. Hyde C, Rigoli D, Piek J. Developmental coordination disorder. In: Rinehart NJ, Bradshaw JL, Enticott PG, editors. Developmental disorders of the brain. New York, NY: Routledge (2016). p. 63-80.

14. Wilson PH, Smits-Engelsman B, Caeyenberghs K, Steenbergen B, Sugden D, Clark J, et al. Cognitive and neuroimaging findings in developmental coordination disorder: new insights from a systematic review of recent research. Dev Med Child Neurol (2017) 59(11):1117-29. doi:10.1111/dmcn.13530 
15. Elzamarany E, Afifi L, El-Fayoumy NM, Salah H, Nada M. Motor cortex rTMS improves dexterity in relapsing-remitting and secondary progressive multiple sclerosis. Acta Neurol Belg (2016) 116(2):145-50. doi:10.1007/ s13760-015-0540-y

16. Enticott PG, Rinehart NJ, Tonge BJ, Bradshaw JL, Fitzgerald PB. Repetitive transcranial magnetic stimulation (rTMS) improves movement-related cortical potentials in autism spectrum disorders. Brain Stimulat (2012) 5(1):30-7. doi:10.1016/j.brs.2011.02.001

17. Lüdemann-Podubecká J, Bösl K, Theilig S, Wiederer R, Nowak DA. The effectiveness of $1 \mathrm{~Hz}$ rTMS over the primary motor area of the unaffected hemisphere to improve hand function after stroke depends on hemispheric dominance. Brain Stimulat (2015) 8(4):823-30. doi:10.1016/j.brs.2015.02.004

18. Sasaki N, Abo M, Hara T, Yamada N, Niimi M, Kakuda W. High-frequency rTMS on leg motor area in the early phase of stroke. Acta Neurol Belg (2017) 117(1):189-94. doi:10.1007/s13760-016-0687-1

19. Stinear CM, Coxon JP, Byblow WD. Primary motor cortex and movement prevention: where stop meets go. Neurosci Biobehav Rev (2009) 33(5):662-73. doi:10.1016/j.neubiorev.2008.08.013

20. Aron AR. From reactive to proactive and selective control: developing a richer model for stopping inappropriate responses. Biol Psychiatry (2011) 69(12):e55-68. doi:10.1016/j.biopsych.2010.07.024

21. MacDonald HJ, Coxon JP, Stinear CM, Byblow WD. The fall and rise of corticomotor excitability with cancellation and reinitiation of prepared action. J Neurophysiol (2014) 112(11):2707-17. doi:10.1152/jn.00366. 2014

22. Beck S, Hallett M. Surround inhibition in the motor system. Exp Brain Res (2011) 210(2):165-72. doi:10.1007/s00221-011-2610-6

23. Byblow WD, Stinear CM. Modulation of short-latency intracortical inhibition in human primary motor cortex during synchronised versus syncopated finger movements. Exp Brain Res (2006) 168(1-2):287-93. doi:10.1007/ s00221-005-0205-9

24. Cowie MJ, MacDonald HJ, Cirillo J, Byblow WD. Proactive modulation of long-interval intracortical inhibition during response inhibition. J Neurophysiol (2016) 116(2):859-67. doi:10.1152/jn.00144.2016

25. Coxon JP, Stinear CM, Byblow WD. Intracortical inhibition during volitional inhibition of prepared action. J Neurophysiol (2006) 95(6):3371-83. doi:10.1152/jn.01334.2005

26. Reynolds C, Ashby P. Inhibition in the human motor cortex is reduced just before a voluntary contraction. Neurology (1999) 53(4):730-5. doi:10.1212/ WNL.53.4.730

27. Capaday C. The integrated nature of motor cortical function. Neuroscientist (2004) 10(3):207-20. doi:10.1177/107385403262109

28. Liepert J, Classen J, Cohen LG, Hallett M. Task-dependent changes of intracortical inhibition. Exp Brain Res (1998) 118(3):421-6. doi:10.1007/ s002210050296

29. Schneider C, Devanne H, Lavoie BA, Capaday C. Neural mechanisms involved in the functional linking of motor cortical points. Exp Brain Res (2002) 146(1):86-94. doi:10.1007/s00221-002-1137-2

30. Beck S, Richardson SP, Shamim EA, Dang N, Schubert M, Hallett M. Short intracortical and surround inhibition are selectively reduced during movement initiation in focal hand dystonia. JNeurosci (2008) 28(41): 10363-9. doi:10.1523/JNEUROSCI.3564-08.2008

31. Beck S, Shamim E, Pirio Richardson S, Schubert M, Hallett M. Interhemispheric inhibition is impaired in mirror dystonia. Eur J Neurosci (2009) 29(8):1634-40. doi:10.1111/j.1460-9568.2009.06710.x

32. Leocani L, Cohen LG, Wassermann EM, Ikoma K, Hallett M. Human corticospinal excitability evaluated with transcranial magnetic stimulation during different reaction time paradigms. Brain (2000) 123(Pt 6):1161-73. doi:10.1093/brain/123.6.1161

33. Sohn YH, Wiltz K, Hallett M. Effect of volitional inhibition on cortical inhibitory mechanisms. JNeurophysiol (2002) 88(1):333. doi:10.1152/ jn.2002.88.1.333

34. Aron AR, Poldrack RA. The cognitive neuroscience of response inhibition: relevance for genetic research in attention-deficit/hyperactivity disorder. Biol Psychiatry (2005) 57(11):1285-92. doi:10.1016/j.biopsych.2004. 10.026

35. Beaulé V, Tremblay S, Théoret $\mathrm{H}$. Interhemispheric control of unilateral movement. Neural Plast (2012) 2012:627816-627816. doi:10.1155/2012/ 627816
36. Swinnen SP, Young DE, Walter CB, Serrien DJ. Control of asymmetrical bimanual movements. Exp Brain Res (1991) 85(1):163-73. doi:10.1007/ BF00229998

37. LiJ-Y, Espay AJ, Gunraj CA, Pal PK, Cunic DI, Lang AE, et al. Interhemispheric and ipsilateral connections in Parkinson's disease: relation to mirror movements. Mov Disord (2007) 22(6):813-21. doi:10.1002/mds.21386

38. Nadkarni NA, Deshmukh SS. Mirror movements. Ann Indian Acad Neurol (2012) 15(1):13. doi:10.4103/0972-2327.93268

39. Cincotta M, Borgheresi A, Balestrieri F, Giovannelli F, Ragazzoni A, Vanni P, et al. Mechanisms underlying mirror movements in Parkinson's disease: a transcranial magnetic stimulation study. Mov Disord (2006) 21(7): 1019-25. doi:10.1002/mds.20850

40. Cox BC, Cincotta M, Espay AJ. Mirror movements in movement disorders: a review. Tremor Other Hyperkinet Mov (N Y) (2012) 2. doi:10.7916/ D8VQ31DZ

41. Spagnolo F, Coppi E, Chieffo R, Straffi L, Fichera M, Nuara A, et al. Interhemispheric balance in Parkinson's disease: a transcranial magnetic stimulation study. Brain Stimulat (2013) 6(6):892-7. doi:10.1016/j.brs.2013. 05.004

42. Vidal J, Derkinderen P, Vidailhet M, Thobois S, Broussolle E. Mirror movements of the non-affected hand in hemiparkinsonian patients: a reflection of ipsilateral motor overactivity? J Neurol Neurosurg Psychiatry (2003) 74(9):1352-3. doi:10.1136/jnnp.74.9.1352

43. Berardelli A, Abbruzzese G, Chen R, Orth M, Ridding MC, Stinear C, et al. Consensus paper on short-interval intracortical inhibition and other transcranial magnetic stimulation intracortical paradigms in movement disorders. Brain Stimulat (2008) 1(3):183-91. doi:10.1016/j.brs.2008. 06.005

44. Heise K-F, Zimerman M, Hoppe J, Gerloff C, Wegscheider K, Hummel FC. The aging motor system as a model for plastic changes of GABA-mediated intracortical inhibition and their behavioral relevance. JNeurosci (2013) 33(21):9039-49. doi:10.1523/JNEUROSCI.4094-12.2013

45. Gilbert D, Isaacs K, Augusta M, Macneil L, Mostofsky S. Motor cortex inhibition A marker of ADHD behavior and motor development in children. Neurology (2011) 76(7):615-21. doi:10.1212/WNL.0b013e31820c2ebd

46. Berardelli A, Rona S, Inghilleri M, Manfredi M. Cortical inhibition in Parkinson's disease. Brain (1996) 119(1):71-7. doi:10.1093/brain/119.1.71

47. Ridding $M$, Rothwell J, Inzelberg R. Changes in excitability of motor cortical circuitry in patients with Parkinson's disease. Ann Neurol (1995) 37(2):181-8. doi:10.1002/ana.410370208

48. Abbruzzese G, Buccolieri A, Marchese R, Trompetto C, Mandich P, Schieppati M. Intracortical inhibition and facilitation are abnormal in Huntington's disease: a paired magnetic stimulation study. Neurosci Lett (1997) 228(2):87-90. doi:10.1016/S0304-3940(97)00363-7

49. Philpott AL, Cummins TDR, Bailey NW, Churchyard A, Fitzgerald PB, Georgiou-Karistianis N. Cortical inhibitory deficits in premanifest and early Huntington's disease. Behav Brain Res (2016) 296:311-7. doi:10.1016/j. bbr.2015.09.030

50. Stinear CM, Byblow WD. Elevated threshold for intracortical inhibition in focal hand dystonia. Mov Disord (2004) 19(11):1312-7. doi:10.1002/ mds. 20160

51. Stinear CM, Byblow WD. Impaired modulation of intracortical inhibition in focal hand dystonia. Cereb Cortex (2004) 14(5):555-61. doi:10.1093/cercor/ bhh017

52. Heise K-F, Steven B, Liuzzi G, Thomalla G, Jonas M, Müller-Vahl K, et al. Altered modulation of intracortical excitability during movement preparation in Gilles de la Tourette syndrome. Brain (2009) 133(2):580-90. doi:10.1093/brain/awp299

53. Bunse T, Wobrock T, Strube W, Padberg F, Palm U, Falkai P, et al. Motor cortical excitability assessed by transcranial magnetic stimulation in psychiatric disorders: a systematic review. Brain Stimulat (2014) 7(2):158-69. doi:10.1016/j.brs.2013.08.009

54. Taylor SF, Tso IF. GABA abnormalities in schizophrenia: a methodological review of in vivo studies. Schizophr Res (2015) 167(1):84-90. doi:10.1016/j. schres.2014.10.011

55. Reis J, Swayne OB, Vandermeeren Y, Camus M, Dimyan MA, Harris-Love M, et al. Contribution of transcranial magnetic stimulation to the understanding of cortical mechanisms involved in motor control. J Physiol (2008) 586(2):325-51. doi:10.1113/jphysiol.2007.144824 
56. Tremblay S, Beaulé V, Proulx S, De Beaumont L, Marjańska M, Doyon J, et al. Relationship between transcranial magnetic stimulation measures of intracortical inhibition and spectroscopy measures of GABA and glutamate+ glutamine. J Neurophysiol (2013) 109(5):1343-9. doi:10.1152/jn.00704.2012

57. Werhahn KJ, Kunesch E, Noachtar S, Benecke R, Classen J. Differential effects on motorcortical inhibition induced by blockade of GABA uptake in humans. J Physiol (1999) 517(2):591-7. doi:10.1111/j.1469-7793.1999.0591t.x

58. Ilić TV, Meintzschel F, Cleff U, Ruge D, Kessler KR, Ziemann U. Short-interval paired-pulse inhibition and facilitation of human motor cortex: the dimension of stimulus intensity. J Physiol (2002) 545(1):153-67. doi:10.1113/jphysiol.2002.030122

59. McDonnell MN, Orekhov Y, Ziemann U. The role of GABAB receptors in intracortical inhibition in the human motor cortex. Exp Brain Res (2006) 173(1):86-93. doi:10.1007/s00221-006-0365-2

60. Inghilleri M, Berardelli A, Cruccu G, Manfredi M. Silent period evoked by transcranial stimulation of the human cortex and cervicomedullary junction. J Physiol (1993) 466:521.

61. Orth M, Rothwell JC. The cortical silent period: intrinsic variability and relation to the waveform of the transcranial magnetic stimulation pulse. Neurophysiol Clin (2004) 115(5):1076-82. doi:10.1016/j.clinph.2003.12.025

62. Ziemann U, Lönnecker S, Steinhoff BJ, Paulus W. The effect of lorazepam on the motor cortical excitability in man. Exp Brain Res (1996) 109(1):127-35. doi:10.1007/BF00228633

63. Di Lazzaro V, Oliviero A, Profice P, Insola A, Mazzone P, Tonali P, et al. Direct demonstration of interhemispheric inhibition of the human motor cortex produced by transcranial magnetic stimulation. Exp Brain Res (1999) 124(4):520-4. doi: 10.1007/s002210050648

64. Ferbert A, Priori A, Rothwell J, Day B, Colebatch J, Marsden C. Interhemispheric inhibition of the human motor cortex. J Physiol (1992) 453(1): 525-46. doi:10.1113/jphysiol.1992.sp019243

65. Perez MA, Cohen LG. Interhemispheric inhibition between primary motor cortices: what have we learned? J Physiol (2009) 587(4):725-6. doi:10.1113/ jphysiol.2008.166926

66. Giovannelli F, Borgheresi A, Balestrieri F, Zaccara G, Viggiano MP, Cincotta M, et al. Modulation of interhemispheric inhibition by volitional motor activity: an ipsilateral silent period study. J Physiol (2009) 587(22):5393-410. doi:10.1113/jphysiol.2009.175885

67. Lo Y, Fook-Chong S. A transcranial magnetic stimulation study of the ipsilateral silent period in lower limb muscles. Neurosci Lett (2004) 368(3):337-40. doi:10.1016/j.neulet.2004.07.080

68. Wassermann EM, Fuhr P, Cohen LG, Hallett M. Effects of transcranial magnetic stimulation on ipsilateral muscles. Neurology (1991) 41(11): 1795-1795. doi:10.1212/WNL.41.11.1795

69. Arányi Z, Rösler KM. Effort-induced mirror movements. Exp Brain Res (2002) 145(1):76-82. doi:10.1007/s00221-002-1101-1

70. Kanouchi T, Yokota T, Isa F, Ishii K, Senda M. Role of the ipsilateral motor cortex in mirror movements. J Neurol Neurosurg Psychiatry (1997) 62(6):629-32. doi:10.1136/jnnp.62.6.629

71. Ueki Y, Mima T, Oga T, Ikeda A, Hitomi T, Fukuyama H, et al. Dominance of ipsilateral corticospinal pathway in congenital mirror movements. J Neurol Neurosurg Psychiatry (2005) 76(2):276-9. doi:10.1136/jnnp.2004. 040949

72. Irlbacher K, Brocke J, Mechow J, Brandt SA. Effects of GABA A and GABA B agonists on interhemispheric inhibition in man. Neurophysiol Clin (2007) 118(2):308-16. doi:10.1016/j.clinph.2006.09.023

73. Blais M, Amarantini D, Albaret JM, Chaix Y, Tallet J. Atypical interhemispheric communication correlates with altered motor inhibition during learning of a new bimanual coordination pattern in developmental coordination disorder. Dev Sci (2017). doi:10.1111/desc. 12563

74. Blais M, Baly C, Biotteau M, Albaret J-M, Chaix Y, Tallet J. Lack of motor inhibition as a marker of learning difficulties of bimanual coordination in teenagers with developmental coordination disorder. Dev Neuropsychol (2017) 42(3):207-19. doi:10.1080/87565641.2017.1306526

75. Licari M, Larkin D. Increased associated movements: influence of attention deficits and movement difficulties. Hum Mov Sci (2008) 27(2):310-24. doi:10.1016/j.humov.2008.02.013

76. Licari M, Larkin D, Miyahara M. The influence of developmental coordination disorder and attention deficits on associated movements in children. Hum Mov Sci (2006) 25(1):90-9. doi:10.1016/j.humov.2005.10.012
77. Licari MK, Billington J, Reid SL, Wann JP, Elliott CM, Winsor AM, et al. Cortical functioning in children with developmental coordination disorder: a motor overflow study. Exp Brain Res (2015) 233(6):1703-10. doi:10.1007/ s00221-015-4243-7

78. Hyde C, Wilson PH. Dissecting online control in developmental coordination disorder: a kinematic analysis of double-step reaching. Brain Cogn (2011) 75(3):232-41. doi:10.1016/j.bandc.2010.12.004

79. Mandich A, Buckolz E, Polatajko H. Children with developmental coordination disorder (DCD) and their ability to disengage ongoing attentional focus: more on inhibitory function. Brain Cogn (2003) 51(3):346-56. doi:10.1016/ S0278-2626(03)00039-3

80. Querne L, Berquin P, Vernier-Hauvette MP, Fall S, Deltour L, Meyer ME, et al. Dysfunction of the attentional brain network in children with developmental coordination disorder: a fMRI study. Brain Res (2008) 1244:89-102. doi:10.1016/j.brainres.2008.07.066

81. Rahimi-Golkhandan S, Steenbergen B, Piek J, Wilson P. Reprint of 'Deficits of hot executive function in developmental coordination disorder: sensitivity to positive social cues'. Hum Mov Sci (2015) 42:352-67. doi:10.1016/j. humov.2015.06.004

82. Rahimi-Golkhandan S, Steenbergen B, Piek JP, Caeyenberghs K, Wilson PH. Revealing hot executive function in children with motor coordination problems: what's the go? Brain Cogn (2016) 106:55-64. doi:10.1016/j.bandc. 2016.04.010

83. Ruddock S, Caeyenberghs K, Piek J, Sugden D, Hyde C, Morris S, et al. Coupling of online control and inhibitory systems in children with atypical motor development: a growth curve modelling study. Brain Cogn (2016) 109:84-95. doi:10.1016/j.bandc.2016.08.001

84. Ruddock S, Piek J, Sugden D, Morris S, Hyde C, Caeyenberghs K, et al. Coupling online control and inhibitory systems in children with developmental coordination disorder: goal-directed reaching. Res Dev Disabil (2015) 36:244-55. doi:10.1016/j.ridd.2014.10.013

85. Tsai C-L, Pan C-Y, Cherng R-J, Hsu Y-W, Chiu H-H. Mechanisms of deficit of visuospatial attention shift in children with developmental coordination disorder: a neurophysiological measure of the endogenous Posner paradigm. Brain Cogn (2009) 71(3):246-58. doi:10.1016/j.bandc.2009. 08.006

86. Tsai C-L, Yu Y-K, Chen Y-J, Wu S-K. Inhibitory response capacities of bilateral lower and upper extremities in children with developmental coordination disorder in endogenous and exogenous orienting modes. Brain Cogn (2009) 69(2):236-44. doi:10.1016/j.bandc.2008.07.012

87. Wilson PH, Maruff P. Deficits in the endogenous control of covert visuospatial attention in children with developmental coordination disorder. Hum Mov Sci (1999) 18(2):421-42. doi:10.1016/S0167-9457(99)00017-2

88. Piek JP, Dyck MJ, Francis M, Conwell A. Working memory, processing speed, and set-shifting in children with developmental coordination disorder and attention-deficit-hyperactivity disorder. Dev Med Child Neurol (2007) 49(9):678-83. doi:10.1111/j.1469-8749.2007.00678.x

89. Leonard HC, Bernardi M, Hill EL, Henry LA. Executive functioning, motor difficulties, and developmental coordination disorder. Dev Neuropsychol (2015) 40(4):201-15. doi:10.1080/87565641.2014.997933

90. Hyde C, Fuelscher I, Williams J, Lum JAG, He J, Barhoun P, et al. Corticospinal excitability during motor imagery is reduced in young adults with developmental coordination disorder. Res Dev Disabil (2018) 72:214-24. doi:10.1016/j.ridd.2017.11.009

91. Williams J, Kashuk SR, Wilson PH, Thorpe G, Egan GF. White matter alterations in adults with probable developmental coordination disorder: an MRI diffusion tensor imaging study. Neuroreport (2017) 28(2):87-92. doi:10.1097/ WNR.0000000000000711

92. Du W, Wilmut K, Barnett AL. Level walking in adults with and without developmental coordination disorder: an analysis of movement variability. Hum Mov Sci (2015) 43:9-14. doi:10.1016/j.humov.2015.06.010

93. Hyde C, Fuelscher I, Buckthought K, Enticott PG, Gitay MA, Williams J. Motor imagery is less efficient in adults with probable developmental coordination disorder: evidence from the hand rotation task. Res Dev Disabil (2014) 35(11):3062-70. doi:10.1016/j.ridd.2014.07.042

94. Wilmut K, Du W, Barnett AL. How do i fit through that gap? Navigation through apertures in adults with and without developmental coordination disorder. PLoS One (2015) 10(4):e0124695. doi:10.1371/journal. pone. 0124695 
95. Kirby A, Edwards L, Sugden D, Rosenblum S. The development and standardization of the adult developmental co-ordination disorders/dyspraxia checklist (ADC). Res Dev Disabil (2010) 31(1):131-9. doi:10.1016/j.ridd. 2009.08.010

96. Bruininks RH. Bruininks-Oseretsky Test of Motor Proficiency (BOT-2). Circle Pines, MN: AGS Publishing (2005). p. 27-28.

97. Hands B, Licari M, Piek J. A review of five tests to identify motor coordination difficulties in young adults. Res Dev Disabil (2015) 41:40-51. doi:10.1016/j. ridd.2015.05.009

98. McIntyre F, Parker H, Thornton A, Licari M, Piek J, Rigoli D, et al. Assessing motor proficiency in young adults: the Bruininks Oseretsky Test-2 short form and the McCarron assessment of neuromuscular development. Hum Mov Sci (2016) 53:55-62. doi:10.1016/j.humov.2016. 10.004

99. Barnett AL, Hill EL, Kirby A, Sugden DA. Adaptation and extension of the European recommendations (EACD) on developmental coordination disorder (DCD) for the UK context. Phys Occup Ther Pediatr (2015) 35(2):103-15. doi:10.3109/01942638.2014.957430

100. Hyde C, Fuelscher I, Lum JA, Williams J, He J, Enticott PG. Primary motor cortex excitability is modulated during the mental simulation of hand movement. J Int Neuropsychol Soc (2017). 23(2):185-93.

101. Kashuk SR, Williams J, Thorpe G, Wilson PH, Egan GF. Diminished motor imagery capability in adults with motor impairment: an fMRI mental rotation study. Behav Brain Res (2017) 334:86-96. doi:10.1016/j.bbr.2017. 06.042

102. Wilmut K, Byrne M, Barnett AL. Reaching to throw compared to reaching to place: a comparison across individuals with and without developmental coordination disorder. Res Dev Disabil (2013) 34(1):174-82. doi:10.1016/j. ridd.2012.07.020

103. Kumru H, Albu S, Rothwell J, Leon D, Flores C, Opisso E, et al. Modulation of motor cortex excitability by paired peripheral and transcranial magnetic stimulation. Neurophysiol Clin (2017) 128(10):2043-7. doi:10.1016/j. clinph.2017.06.041

104. Rossini PM, Burke D, Chen R, Cohen L, Daskalakis Z, Di Iorio R, et al. Non-invasive electrical and magnetic stimulation of the brain, spinal cord, roots and peripheral nerves: basic principles and procedures for routine clinical and research application. An updated report from an IFCN Committee. Neurophysiol Clin (2015) 126(6):1071-107. doi:10.1016/j. clinph.2015.02.001

105. Orth M, Snijders A, Rothwell J. The variability of intracortical inhibition and facilitation. Neurophysiol Clin (2003) 114(12):2362-9. doi:10.1016/ S1388-2457(03)00243-8

106. Ziemann U, Rothwell JC, Ridding MC. Interaction between intracortical inhibition and facilitation in human motor cortex. JPhysiol (1996) 496(Pt 3):873. doi:10.1113/jphysiol.1996.sp021734

107. Daskalakis ZJ, Farzan F, Barr MS, Maller JJ, Chen R, Fitzgerald PB. Longinterval cortical inhibition from the dorsolateral prefrontal cortex: a TMSEEG study. Neuropsychopharmacology (2008) 33(12):2860. doi:10.1038/ npp.2008.22

108. Enticott PG, Kennedy HA, Rinehart NJ, Tonge BJ, Bradshaw JL, Fitzgerald PB. GABAergic activity in autism spectrum disorders: an investigation of cortical inhibition via transcranial magnetic stimulation. Neuropharmacology (2013) 68:202-9. doi:10.1016/j.neuropharm.2012.06.017

109. Sanger TD, Garg RR, Chen R. Interactions between two different inhibitory systems in the human motor cortex. J Physiol (2001) 530(2):307-17. doi:10.1111/j.1469-7793.2001.0307l.x
110. Cantello R, Gianelli M, Civardi C, Mutani R. Magnetic brain stimulation the silent period after the motor evoked potential. Neurology (1992) 42(10):1951-1951. doi:10.1212/WNL.42.10.1951

111. Kuo Y-L, Dubuc T, Boufadel DF, Fisher BE. Measuring ipsilateral silent period: effects of muscle contraction levels and quantification methods. Brain Res (2017) 1674(Suppl C):77-83. doi:10.1016/j.brainres.2017.08.015

112. Jung P, Ziemann U. Differences of the ipsilateral silent period in small hand muscles. Muscle Nerve (2006) 34(4):431-6. doi:10.1002/mus.20604

113. Garvey MA, Ziemann U, Becker DA, Barker CA, Bartko JJ. New graphical method to measure silent periods evoked by transcranial magnetic stimulation. Neurophysiol Clin (2001) 112(8):1451-60. doi:10.1016/S13882457(01)00581-8

114. Chen R, Yung D, Li JY. Organization of ipsilateral excitatory and inhibitory pathways in the human motor cortex. J Neurophysiol (2003) 89(3):1256-64. doi:10.1152/jn.00950.2002

115. Gilbert DL, Bansal AS, Sethuraman G, Sallee FR, Zhang J, Lipps T, et al. Association of cortical disinhibition with tic, ADHD, and OCD severity in Tourette syndrome. Mov Disord (2004) 19(4):416-25. doi:10.1002/mds.20044

116. Waldvogel D, van Gelderen P, Muellbacher W, Ziemann U, Immisch I, Hallett $M$. The relative metabolic demand of inhibition and excitation. Nature (2000) 406(6799):995-8. doi:10.1038/35023171

117. Meyer BU, Röricht S, Von Einsiedel HG, Kruggel F, Weindl A. Inhibitory and excitatory interhemispheric transfers between motor cortical areas in normal humans and patients with abnormalities of the corpus callosum. Brain (1995) 118(2):429-40. doi:10.1093/brain/118.2.429

118. Duque J, Murase N, Celnik P, Hummel F, Harris-Love M, Mazzocchio R, et al. Intermanual differences in movement-related interhemispheric inhibition. J Cogn Neurosci (2007) 19(2):204-13. doi:10.1162/jocn.2007.19.2.204

119. Tallet J, Albaret J-M, Barral J. Developmental changes in lateralized inhibition of symmetric movements in children with and without developmental coordination disorder. Res Dev Disabil (2013) 34(9):2523-32. doi:10.1016/j. ridd.2013.05.020

120. Langevin LM, MacMaster FP, Crawford S, Lebel C, Dewey D. Common white matter microstructure alterations in pediatric motor and attention disorders. J Pediatr (2014) 164(5):1157-64.e1. doi:10.1016/j.jpeds.2014.01.018

121. Bäumer T, Dammann E, Bock F, Klöppel S, Siebner HR, Münchau A. Laterality of interhemispheric inhibition depends on handedness. Exp Brain Res (2007) 180(2):195-203. doi:10.1007/s00221-007-0866-7

122. Fitzgerald PB, Fountain S, Daskalakis ZJ. A comprehensive review of the effects of rTMS on motor cortical excitability and inhibition. Neurophysiol Clin (2006) 117(12):2584-96. doi:10.1016/j.clinph.2006.06.712

123. Nettekoven C, Volz LJ, Kutscha M, Pool E-M, Rehme AK, Eickhoff SB, et al. Dose-dependent effects of theta burst rTMS on cortical excitability and resting-state connectivity of the human motor system. J Neurosci (2014) 34(20):6849-59. doi:10.1523/JNEUROSCI.4993-13.2014

Conflict of Interest Statement: The authors declare that the research was conducted in the absence of any commercial or financial relationships that could be construed as a potential conflict of interest.

Copyright $\odot 2018$ He, Fuelscher, Enticott, Teo, Barhoun and Hyde. This is an open-access article distributed under the terms of the Creative Commons Attribution License (CC BY). The use, distribution or reproduction in other forums is permitted, provided the original author(s) and the copyright owner are credited and that the original publication in this journal is cited, in accordance with accepted academic practice. No use, distribution or reproduction is permitted which does not comply with these terms. 